East Asian Math. J.

Vol. 30 (2014), No.3, pp. 361-369

http://dx.doi.org/10.7858/eamj.2014.026

\title{
CHARACTERIZATIONS OF FUNCTIONS ON DUAL OCTONION VARIABLES IN CLIFFORD ANALYSIS
}

\author{
Su Mi Lim And Kwang Ho SHON*
}

\begin{abstract}
The aim of this paper is to define hyperholomorphic functions with dual octonion variables in $\mathbb{C}^{4} \times \mathbb{C}^{4}$. We characterize properties of dual octonion variables in Clifford analysis.
\end{abstract}

\section{Introduction}

The octonions are a normed division algebra over the real numbers in Clifford algebra. There are four such algebras, the other three being the real numbers $\mathbb{R}$, the complex numbers $\mathbb{C}$, and the quaternions $\mathbb{H}$. Octonions are an extension of quaternions which are double the number. In addition, octonions satisfy noncommutative and nonassociative, however, they satisfy a weaker form of associativity which is alternative. Even though octonions are not that famous compared to the quaternions and complex numbers which are much more studied and used, they have some interesting properties and are related to a number of exceptional structures in mathematics. Additionally, octonions have applications in fields such as string theory, special relativity theory, and quantum logic. The octonions were invented in 1843 by John T. Graves, inspired by William Hamilton's discovery of quaternions. Graves called his discovery octaves. In 1973, Deutoni and Sce [1] defined octonionic regular functions and some properties of octonionc regular functions. And K. Nôno [46] found some properties of hyperholomorphic functions. In 2013, Lim and Shon [3] researched the properties of hyperholomorphic functions and obtained the properties of corresponding o-Cauchy-Riemann equation with octonion variables in $\mathbb{C}^{4}$. The function $g(z)=g_{1}(z)+g_{2}(z) e_{2}+g_{3}(z) e_{4}+g_{4}(z) e_{6}$ where $z=\left(z_{1}, z_{2}, z_{3}, z_{4}\right)$ and the functions $g_{1}(z), g_{2}(z), g_{3}(z)$ and $g_{4}(z)$ are harmonic in $\Omega$ satisfies the condition of harmonicity in an open set $\Omega$ in $\mathbb{C}^{4}$. Besides, we [2] found the theorem about hyperholomorphic functions of dual quaternion in an open subset of $\mathbb{C}^{2} \times \mathbb{C}^{2}$.

Received April 15, 2014; Accepted May 29, 2014.

2010 Mathematics Subject Classification. 32A99, 30G35, 11E88.

Key words and phrases. Clifford Analysis, hyperholomorphic function, octonion variables, dual number system, the corresponding o-Cauchy-Riemann equation.

*Corresponding author.

(C)2014 The Youngnam Mathematical Society (pISSN 1226-6973, eISSN 2287-2833) 
In this paper, we investigate the properties of hyperholomorphic functions of dual octonion variables and find the condition of integrability of the corresponding o-Cauchy-Riemann equation in an open subset of $\mathbb{C}^{4} \times \mathbb{C}^{4}$.

\section{Preliminaries}

The field $\mathcal{O} \cong \mathbb{C}^{4}$ of octonions

$$
z=x_{0}+\sum_{j=0}^{7} e_{j} x_{j}, \quad\left(x_{j} \in \mathbb{R}, j=0, \cdots, 7\right)
$$

is an eight dimensional non-commutative and non-associative $\mathbb{R}$-field generated by eight base elements $e_{0}, e_{1}, e_{2}, e_{3}, e_{4}, e_{5}, e_{6}$ and $e_{7}$ with the following noncommutative multiplication rules :

$$
\begin{aligned}
& e_{i}^{2}=-1, e_{i} e_{j}=-e_{j} e_{i}, e_{i} e_{j} e_{k}=e_{i}\left(e_{j} e_{k}\right)(i \neq j \neq k, i \neq 0, j \neq 0, k \neq 0) \\
& e_{1} e_{2}=e_{3}, e_{3} e_{5}=e_{6}, e_{6} e_{7}=e_{1}, e_{1} e_{4}=e_{5}, e_{5} e_{7}=e_{2}, e_{2} e_{6}=e_{4}, e_{4} e_{7}=e_{3} .
\end{aligned}
$$

The element $e_{0}$ is the identities of $\mathcal{O}$ and $e_{1}$ identify the imaginary unit $\sqrt{-1}$ in the $\mathbb{C}$-field of complex numbers. An octonion $z$ given by (1) is regarded as $z=z_{1}+z_{2} e_{2}+z_{3} e_{4}+z_{4} e_{6} \in \mathcal{O}$ where $z_{1}:=x_{0}+e_{1} x_{1}, z_{2}:=x_{2}+e_{1} x_{3}$, $z_{3}:=x_{4}+e_{1} x_{5}$ and $z_{4}:=x_{6}+e_{1} x_{7}$ are complex numbers in $\mathbb{C}$. Thus, we identify $\mathcal{O}$ with $\mathbb{C}^{4}$. the octonionic conjugation $z^{*}$, the absolute value $|z|$ of $z$ and a inverse $z^{-1}$ of $\mathrm{z}$ in $\mathcal{O}$ are defined, respectively, by

$$
z^{*}=x_{0}-\sum_{j=1}^{7} e_{j} x_{j},|z|=\sqrt{\sum_{j=1}^{4}\left|z_{j}\right|^{2}}, z^{-1}=\frac{z^{*}}{|z|^{2}}(z \neq 0) .
$$

\section{Some properties of hyperholomorphic functions on dual octonion variables}

The dual numbers extended the real numbers by adjoining one new element $\varepsilon$ with the property $\varepsilon^{2}=0$. Every dual number has the form $z=x+\varepsilon y$ with $x$ and $y$ uniquely determined real numbers. And the conjugate dual number $z^{*}$ of $z$ is defined by $z^{*}=x-\varepsilon y$ and we obtain $|z|^{2}=x^{2}$. If we use matrices, dual numbers can be represented as

$$
\varepsilon=\left(\begin{array}{ll}
0 & 1 \\
0 & 0
\end{array}\right), z=x+\varepsilon y=\left(\begin{array}{ll}
x & y \\
0 & x
\end{array}\right) .
$$

The dual numbers are elements of the 2-dimensional real algebra

$$
B=\left\{z=x+\varepsilon y \mid x, y \in \mathbb{R}, \varepsilon^{2}=0\right\}
$$

generated by 1 and $\varepsilon$. The dual octonion $z=\sum_{j=0}^{7} e_{j} x_{j}+\varepsilon \sum_{j=0}^{7} e_{j} y_{j}$ of $B$ is written as $z=a+\varepsilon b$. The conjugation number $z^{*}$, the absolute value $|z|$ and 
the inverse $z^{-1}$ of $z=a+\varepsilon b$ are given by the followings:

$$
\begin{aligned}
& z^{*}=x_{0}-\sum_{j=1}^{7} e_{j} x_{j}+\varepsilon\left(y_{0}-\sum_{j=1}^{7} e_{j} y_{j}\right)=a^{*}+\varepsilon b^{*}, \\
& |z|^{2}=z z^{*}=\sum_{j=0}^{7} x_{j}{ }^{2}+2 \varepsilon \sum_{j=0}^{7} x_{j} y_{j}=\sum_{j=0}^{7} \zeta_{j}^{2}, z^{-1}=\frac{z^{*}}{|z|^{2}},
\end{aligned}
$$

where $\zeta_{j}=x_{j}+\varepsilon y_{j}(j=0, \cdots, 7), a^{*}$ and $b^{*}$ are conjugate numbers of $a$ and $b$, respectively. We consider the following differential operators:

$$
\begin{aligned}
& D:=\frac{1}{2}\left(\frac{\partial}{\partial x_{0}}-e_{1} \frac{\partial}{\partial x_{1}}-e_{2} \frac{\partial}{\partial x_{2}}+e_{3} \frac{\partial}{\partial x_{3}}-e_{4} \frac{\partial}{\partial x_{4}}+e_{5} \frac{\partial}{\partial x_{5}}-e_{6} \frac{\partial}{\partial x_{6}}+e_{7} \frac{\partial}{\partial x_{7}}\right. \\
& \left.+\varepsilon\left(\frac{\partial}{\partial y_{0}}-e_{1} \frac{\partial}{\partial y_{1}}-e_{2} \frac{\partial}{\partial y_{2}}+e_{3} \frac{\partial}{\partial y_{3}}-e_{4} \frac{\partial}{\partial y_{4}}+e_{5} \frac{\partial}{\partial y_{5}}-e_{6} \frac{\partial}{\partial y_{6}}+e_{7} \frac{\partial}{\partial y_{7}}\right)\right), \\
& D^{*}=\frac{1}{2}\left(\frac{\partial}{\partial x_{0}}+e_{1} \frac{\partial}{\partial x_{1}}+e_{2} \frac{\partial}{\partial x_{2}}-e_{3} \frac{\partial}{\partial x_{3}}+e_{4} \frac{\partial}{\partial x_{4}}-e_{5} \frac{\partial}{\partial x_{5}}+e_{6} \frac{\partial}{\partial x_{6}}-e_{7} \frac{\partial}{\partial x_{7}}\right. \\
& \left.+\varepsilon\left(\frac{\partial}{\partial y_{0}}+e_{1} \frac{\partial}{\partial y_{1}}+e_{2} \frac{\partial}{\partial y_{2}}-e_{3} \frac{\partial}{\partial y_{3}}+e_{4} \frac{\partial}{\partial y_{4}}-e_{5} \frac{\partial}{\partial y_{5}}+e_{6} \frac{\partial}{\partial y_{6}}-e_{7} \frac{\partial}{\partial y_{7}}\right)\right) .
\end{aligned}
$$

Then, we have the following for dual quaternion operators:

$$
D D^{*}=D^{*} D=\frac{1}{4}\left(\sum_{j=0}^{7} \frac{\partial^{2}}{\partial x_{j}{ }^{2}}+2 \varepsilon\left(\sum_{j=0}^{7} \frac{\partial^{2}}{\partial x_{j} \partial y_{j}}\right)\right)=\frac{1}{4}\left(\sum_{j=0}^{7} \frac{\partial^{2}}{\partial \tau_{j}{ }^{2}}\right)=\frac{1}{4} \Delta_{z},
$$

where $\frac{\partial}{\partial \tau_{j}}=\frac{\partial}{\partial x_{j}}+\varepsilon \frac{\partial}{\partial y_{j}}(j=0,1, \cdots, 7)$.

Definition 2. Let $\Omega$ be an open subset of $\mathbb{C}^{4} \times \mathbb{C}^{4}$. A function $F(z)=$ $f(a)+\varepsilon g(b)=\sum_{j=0}^{7} e_{j} u_{j}(a)+\varepsilon\left(\sum_{j=0}^{7} e_{j} v_{j}(b)\right)$ is said to be hyperholomorphic on $\Omega$ if

(1) $u_{j}(a)$ and $v_{j}(b)(j=0,1, \cdots, 7)$ are continuously differentiable on $\Omega$,

(2) $D^{*} F(z)=0$ on $\Omega$.

Equation (2) of Definition 2 operates to $F(z)$ as follows:

$$
\begin{aligned}
2 D^{*} F= & \frac{\partial u_{0}}{\partial x_{0}}-\frac{\partial u_{1}}{\partial x_{1}}-\frac{\partial u_{2}}{\partial x_{2}}+\frac{\partial u_{3}}{\partial x_{3}}-\frac{\partial u_{4}}{\partial x_{4}}+\frac{\partial u_{5}}{\partial x_{5}}-\frac{\partial u_{6}}{\partial x_{6}}+\frac{\partial u_{7}}{\partial x_{7}} \\
& +e_{1}\left(\frac{\partial u_{1}}{\partial x_{0}}+\frac{\partial u_{0}}{\partial x_{1}}+\frac{\partial u_{3}}{\partial x_{2}}+\frac{\partial u_{2}}{\partial x_{3}}+\frac{\partial u_{5}}{\partial x_{4}}+\frac{\partial u_{4}}{\partial x_{5}}+\frac{\partial u_{7}}{\partial x_{6}}+\frac{\partial u_{6}}{\partial x_{7}}\right) \\
& +e_{2}\left(\frac{\partial u_{2}}{\partial x_{0}}-\frac{\partial u_{3}}{\partial x_{1}}+\frac{\partial u_{0}}{\partial x_{2}}-\frac{\partial u_{1}}{\partial x_{3}}-\frac{\partial u_{6}}{\partial x_{4}}-\frac{\partial u_{7}}{\partial x_{5}}+\frac{\partial u_{4}}{\partial x_{6}}+\frac{\partial u_{5}}{\partial x_{7}}\right) \\
& +e_{3}\left(\frac{\partial u_{3}}{\partial x_{0}}+\frac{\partial u_{2}}{\partial x_{1}}-\frac{\partial u_{1}}{\partial x_{2}}-\frac{\partial u_{0}}{\partial x_{3}}+\frac{\partial u_{7}}{\partial x_{4}}-\frac{\partial u_{6}}{\partial x_{5}}-\frac{\partial u_{5}}{\partial x_{6}}+\frac{\partial u_{4}}{\partial x_{7}}\right)
\end{aligned}
$$




$$
\begin{aligned}
& +e_{4}\left(\frac{\partial u_{4}}{\partial x_{0}}-\frac{\partial u_{5}}{\partial x_{1}}+\frac{\partial u_{6}}{\partial x_{2}}+\frac{\partial u_{7}}{\partial x_{3}}+\frac{\partial u_{0}}{\partial x_{4}}-\frac{\partial u_{1}}{\partial x_{5}}-\frac{\partial u_{2}}{\partial x_{6}}-\frac{\partial u_{3}}{\partial x_{7}}\right) \\
& +e_{5}\left(\frac{\partial u_{5}}{\partial x_{0}}+\frac{\partial u_{4}}{\partial x_{1}}-\frac{\partial u_{7}}{\partial x_{2}}+\frac{\partial u_{6}}{\partial x_{3}}-\frac{\partial u_{1}}{\partial x_{4}}-\frac{\partial u_{0}}{\partial x_{5}}+\frac{\partial u_{3}}{\partial x_{6}}-\frac{\partial u_{2}}{\partial x_{7}}\right) \\
& +e_{6}\left(\frac{\partial u_{6}}{\partial x_{0}}-\frac{\partial u_{7}}{\partial x_{1}}-\frac{\partial u_{4}}{\partial x_{2}}-\frac{\partial u_{5}}{\partial x_{3}}+\frac{\partial u_{2}}{\partial x_{4}}+\frac{\partial u_{3}}{\partial x_{5}}+\frac{\partial u_{0}}{\partial x_{6}}-\frac{\partial u_{1}}{\partial x_{7}}\right) \\
& +e_{7}\left(\frac{\partial u_{7}}{\partial x_{0}}+\frac{\partial u_{6}}{\partial x_{1}}+\frac{\partial u_{5}}{\partial x_{2}}-\frac{\partial u_{4}}{\partial x_{3}}-\frac{\partial u_{3}}{\partial x_{4}}+\frac{\partial u_{2}}{\partial x_{5}}-\frac{\partial u_{1}}{\partial x_{6}}-\frac{\partial u_{0}}{\partial x_{7}}\right) \\
& +\varepsilon\left(\frac{\partial v_{0}}{\partial x_{0}}-\frac{\partial v_{1}}{\partial x_{1}}-\frac{\partial v_{2}}{\partial x_{2}}+\frac{\partial v_{3}}{\partial x_{3}}-\frac{\partial v_{4}}{\partial x_{4}}+\frac{\partial v_{5}}{\partial x_{5}}-\frac{\partial v_{6}}{\partial x_{6}}+\frac{\partial v_{7}}{\partial x_{7}}\right. \\
& \left.+\frac{\partial u_{0}}{\partial y_{0}}-\frac{\partial u_{1}}{\partial y_{1}}-\frac{\partial u_{2}}{\partial y_{2}}+\frac{\partial u_{3}}{\partial y_{3}}-\frac{\partial u_{4}}{\partial y_{4}}+\frac{\partial u_{5}}{\partial y_{5}}-\frac{\partial u_{6}}{\partial y_{6}}+\frac{\partial u_{7}}{\partial y_{7}}\right) \\
& +e_{1}\left(\frac{\partial v_{1}}{\partial x_{0}}+\frac{\partial v_{0}}{\partial x_{1}}+\frac{\partial v_{3}}{\partial x_{2}}+\frac{\partial v_{2}}{\partial x_{3}}+\frac{\partial v_{5}}{\partial x_{4}}+\frac{\partial v_{4}}{\partial x_{5}}+\frac{\partial v_{7}}{\partial x_{6}}+\frac{\partial v_{6}}{\partial x_{7}}\right. \\
& \left.+\frac{\partial u_{1}}{\partial y_{0}}+\frac{\partial u_{0}}{\partial y_{1}}+\frac{\partial u_{3}}{\partial y_{2}}+\frac{\partial u_{2}}{\partial y_{3}}+\frac{\partial u_{5}}{\partial y_{4}}+\frac{\partial u_{4}}{\partial y_{5}}+\frac{\partial u_{7}}{\partial y_{6}}+\frac{\partial u_{6}}{\partial y_{7}}\right) \\
& +e_{2}\left(\frac{\partial v_{2}}{\partial x_{0}}-\frac{\partial v_{3}}{\partial x_{1}}+\frac{\partial v_{0}}{\partial x_{2}}-\frac{\partial v_{1}}{\partial x_{3}}-\frac{\partial v_{6}}{\partial x_{4}}-\frac{\partial v_{7}}{\partial x_{5}}+\frac{\partial v_{4}}{\partial x_{6}}+\frac{\partial v_{5}}{\partial x_{7}}\right. \\
& \left.+\frac{\partial u_{2}}{\partial y_{0}}-\frac{\partial u_{3}}{\partial y_{1}}+\frac{\partial u_{0}}{\partial y_{2}}-\frac{\partial u_{1}}{\partial y_{3}}-\frac{\partial u_{6}}{\partial y_{4}}-\frac{\partial u_{7}}{\partial y_{5}}+\frac{\partial u_{4}}{\partial y_{6}}+\frac{\partial u_{5}}{\partial y_{7}}\right) \\
& +e_{3}\left(\frac{\partial v_{3}}{\partial x_{0}}+\frac{\partial v_{2}}{\partial x_{1}}-\frac{\partial v_{1}}{\partial x_{2}}-\frac{\partial v_{0}}{\partial x_{3}}+\frac{\partial v_{7}}{\partial x_{4}}-\frac{\partial v_{6}}{\partial x_{5}}-\frac{\partial v_{5}}{\partial x_{6}}+\frac{\partial v_{4}}{\partial x_{7}}\right. \\
& \left.+\frac{\partial u_{3}}{\partial y_{0}}+\frac{\partial u_{2}}{\partial y_{1}}-\frac{\partial u_{1}}{\partial y_{2}}-\frac{\partial u_{0}}{\partial y_{3}}+\frac{\partial u_{7}}{\partial y_{4}}-\frac{\partial u_{6}}{\partial y_{5}}-\frac{\partial u_{5}}{\partial y_{6}}+\frac{\partial u_{4}}{\partial y_{7}}\right) \\
& +e_{4}\left(\frac{\partial v_{4}}{\partial x_{0}}-\frac{\partial v_{5}}{\partial x_{1}}+\frac{\partial v_{6}}{\partial x_{2}}+\frac{\partial v_{7}}{\partial x_{3}}+\frac{\partial v_{0}}{\partial x_{4}}-\frac{\partial v_{1}}{\partial x_{5}}-\frac{\partial v_{2}}{\partial x_{6}}-\frac{\partial v_{3}}{\partial x_{7}}\right. \\
& \left.+\frac{\partial u_{4}}{\partial y_{0}}-\frac{\partial u_{5}}{\partial y_{1}}+\frac{\partial u_{6}}{\partial y_{2}}+\frac{\partial u_{7}}{\partial y_{3}}+\frac{\partial u_{0}}{\partial y_{4}}-\frac{\partial u_{1}}{\partial y_{5}}-\frac{\partial u_{2}}{\partial y_{6}}-\frac{\partial u_{3}}{\partial y_{7}}\right) \\
& +e_{5}\left(\frac{\partial v_{5}}{\partial x_{0}}+\frac{\partial v_{4}}{\partial x_{1}}-\frac{\partial v_{7}}{\partial x_{2}}+\frac{\partial v_{6}}{\partial x_{3}}-\frac{\partial v_{1}}{\partial x_{4}}-\frac{\partial v_{0}}{\partial x_{5}}+\frac{\partial v_{3}}{\partial x_{6}}-\frac{\partial v_{2}}{\partial x_{7}}\right. \\
& \left.+\frac{\partial u_{5}}{\partial y_{0}}+\frac{\partial u_{4}}{\partial y_{1}}-\frac{\partial u_{7}}{\partial y_{2}}+\frac{\partial u_{6}}{\partial y_{3}}-\frac{\partial u_{1}}{\partial y_{4}}-\frac{\partial u_{0}}{\partial y_{5}}+\frac{\partial u_{3}}{\partial y_{6}}-\frac{\partial u_{2}}{\partial y_{7}}\right) \\
& +e_{6}\left(\frac{\partial v_{6}}{\partial x_{0}}-\frac{\partial v_{7}}{\partial x_{1}}-\frac{\partial v_{4}}{\partial x_{2}}-\frac{\partial v_{5}}{\partial x_{3}}+\frac{\partial v_{2}}{\partial x_{4}}+\frac{\partial v_{3}}{\partial x_{5}}+\frac{\partial v_{0}}{\partial x_{6}}-\frac{\partial v_{1}}{\partial x_{7}}\right. \\
& \left.+\frac{\partial u_{6}}{\partial y_{0}}-\frac{\partial u_{7}}{\partial y_{1}}-\frac{\partial u_{4}}{\partial y_{2}}-\frac{\partial u_{5}}{\partial y_{3}}+\frac{\partial u_{2}}{\partial y_{4}}+\frac{\partial u_{3}}{\partial y_{5}}+\frac{\partial u_{0}}{\partial y_{6}}-\frac{\partial u_{1}}{\partial y_{7}}\right) \\
& +e_{7}\left(\frac{\partial v_{7}}{\partial x_{0}}+\frac{\partial v_{6}}{\partial x_{1}}+\frac{\partial v_{5}}{\partial x_{2}}-\frac{\partial v_{4}}{\partial x_{3}}-\frac{\partial v_{3}}{\partial x_{4}}+\frac{\partial v_{2}}{\partial x_{5}}-\frac{\partial v_{1}}{\partial x_{6}}-\frac{\partial v_{0}}{\partial x_{7}}\right. \\
& \left.+\frac{\partial u_{7}}{\partial y_{0}}+\frac{\partial u_{6}}{\partial y_{1}}+\frac{\partial u_{5}}{\partial y_{2}}-\frac{\partial u_{4}}{\partial y_{3}}-\frac{\partial u_{3}}{\partial y_{4}}+\frac{\partial u_{2}}{\partial y_{5}}-\frac{\partial u_{1}}{\partial y_{6}}-\frac{\partial u_{0}}{\partial y_{7}}\right) .
\end{aligned}
$$


Therefore, the equation (2) of Definition 2 for $F(z)$ is equivalent to the following system of equations:

$$
\begin{aligned}
& \frac{\partial u_{0}}{\partial x_{0}}+\frac{\partial u_{3}}{\partial x_{3}}+\frac{\partial u_{5}}{\partial x_{5}}+\frac{\partial u_{7}}{\partial x_{7}}=\frac{\partial u_{1}}{\partial x_{1}}+\frac{\partial u_{2}}{\partial x_{2}}+\frac{\partial u_{4}}{\partial x_{4}}+\frac{\partial u_{6}}{\partial x_{6}}, \\
& \frac{\partial u_{1}}{\partial x_{0}}+\frac{\partial u_{0}}{\partial x_{1}}+\frac{\partial u_{3}}{\partial x_{2}}+\frac{\partial u_{2}}{\partial x_{3}}+\frac{\partial u_{5}}{\partial x_{4}}+\frac{\partial u_{4}}{\partial x_{5}}+\frac{\partial u_{7}}{\partial x_{6}}+\frac{\partial u_{6}}{\partial x_{7}}=0, \\
& \frac{\partial u_{2}}{\partial x_{0}}+\frac{\partial u_{0}}{\partial x_{2}}+\frac{\partial u_{4}}{\partial x_{6}}+\frac{\partial u_{5}}{\partial x_{7}}=\frac{\partial u_{3}}{\partial x_{1}}+\frac{\partial u_{1}}{\partial x_{3}}+\frac{\partial u_{6}}{\partial x_{4}}+\frac{\partial u_{7}}{\partial x_{5}}, \\
& \frac{\partial u_{3}}{\partial x_{0}}+\frac{\partial u_{2}}{\partial x_{1}}+\frac{\partial u_{7}}{\partial x_{4}}+\frac{\partial u_{4}}{\partial x_{7}}=\frac{\partial u_{1}}{\partial x_{2}}+\frac{\partial u_{0}}{\partial x_{3}}+\frac{\partial u_{6}}{\partial x_{5}}+\frac{\partial u_{5}}{\partial x_{6}}, \\
& \frac{\partial u_{4}}{\partial x_{0}}+\frac{\partial u_{6}}{\partial x_{2}}+\frac{\partial u_{7}}{\partial x_{3}}+\frac{\partial u_{0}}{\partial x_{4}}=\frac{\partial u_{5}}{\partial x_{1}}+\frac{\partial u_{1}}{\partial x_{5}}+\frac{\partial u_{2}}{\partial x_{6}}+\frac{\partial u_{3}}{\partial x_{7}}, \\
& \frac{\partial u_{5}}{\partial x_{0}}+\frac{\partial u_{4}}{\partial x_{1}}+\frac{\partial u_{6}}{\partial x_{3}}+\frac{\partial u_{3}}{\partial x_{6}}=\frac{\partial u_{7}}{\partial x_{2}}+\frac{\partial u_{1}}{\partial x_{4}}+\frac{\partial u_{0}}{\partial x_{5}}+\frac{\partial u_{2}}{\partial x_{7}}, \\
& \frac{\partial u_{6}}{\partial x_{0}}+\frac{\partial u_{2}}{\partial x_{4}}+\frac{\partial u_{3}}{\partial x_{5}}+\frac{\partial u_{0}}{\partial x_{6}}=\frac{\partial u_{3}}{\partial x_{1}}+\frac{\partial u_{4}}{\partial x_{2}}+\frac{\partial u_{5}}{\partial x_{3}}+\frac{\partial u_{1}}{\partial x_{7}}, \\
& \frac{\partial u_{7}}{\partial x_{0}}+\frac{\partial u_{6}}{\partial x_{1}}+\frac{\partial u_{5}}{\partial x_{2}}+\frac{\partial u_{2}}{\partial x_{5}}=\frac{\partial u_{4}}{\partial x_{3}}+\frac{\partial u_{3}}{\partial x_{4}}+\frac{\partial u_{1}}{\partial x_{6}}+\frac{\partial u_{0}}{\partial x_{7}}, \\
& \frac{\partial v_{0}}{\partial x_{0}}+\frac{\partial v_{3}}{\partial x_{3}}+\frac{\partial v_{5}}{\partial x_{5}}+\frac{\partial v_{7}}{\partial x_{7}}+\frac{\partial u_{0}}{\partial y_{0}}+\frac{\partial u_{3}}{\partial y_{3}}+\frac{\partial u_{5}}{\partial y_{5}}+\frac{\partial u_{7}}{\partial y_{7}} \\
& =\frac{\partial v_{1}}{\partial x_{1}}+\frac{\partial v_{2}}{\partial x_{2}}+\frac{\partial v_{4}}{\partial x_{4}}+\frac{\partial v_{6}}{\partial x_{6}}+\frac{\partial u_{1}}{\partial y_{1}}+\frac{\partial u_{2}}{\partial y_{2}}+\frac{\partial u_{4}}{\partial y_{4}}+\frac{\partial u_{6}}{\partial y_{6}}, \\
& \frac{\partial v_{1}}{\partial x_{0}}+\frac{\partial v_{0}}{\partial x_{1}}+\frac{\partial v_{3}}{\partial x_{2}}+\frac{\partial v_{2}}{\partial x_{3}}+\frac{\partial v_{5}}{\partial x_{4}}+\frac{\partial v_{4}}{\partial x_{5}}+\frac{\partial v_{7}}{\partial x_{6}}+\frac{\partial v_{6}}{\partial x_{7}} \\
& +\frac{\partial u_{1}}{\partial y_{0}}+\frac{\partial u_{0}}{\partial y_{1}}+\frac{\partial u_{3}}{\partial y_{2}}+\frac{\partial u_{2}}{\partial y_{3}}+\frac{\partial u_{5}}{\partial y_{4}}+\frac{\partial u_{4}}{\partial y_{5}}+\frac{\partial u_{7}}{\partial y_{6}}+\frac{\partial u_{6}}{\partial y_{7}}=0, \\
& \frac{\partial v_{2}}{\partial x_{0}}+\frac{\partial v_{0}}{\partial x_{2}}+\frac{\partial v_{4}}{\partial x_{6}}+\frac{\partial v_{5}}{\partial x_{7}}+\frac{\partial u_{2}}{\partial y_{0}}+\frac{\partial u_{0}}{\partial y_{2}}+\frac{\partial u_{4}}{\partial y_{6}}+\frac{\partial u_{5}}{\partial y_{7}} \\
& =\frac{\partial v_{3}}{\partial x_{1}}+\frac{\partial v_{1}}{\partial x_{3}}+\frac{\partial v_{6}}{\partial x_{4}}+\frac{\partial v_{7}}{\partial x_{5}}+\frac{\partial u_{3}}{\partial y_{1}}+\frac{\partial u_{1}}{\partial y_{3}}+\frac{\partial u_{6}}{\partial y_{4}}+\frac{\partial u_{7}}{\partial y_{5}}, \\
& \frac{\partial v_{3}}{\partial x_{0}}+\frac{\partial v_{2}}{\partial x_{1}}+\frac{\partial v_{7}}{\partial v_{4}}+\frac{\partial v_{4}}{\partial x_{7}}+\frac{\partial u_{3}}{\partial y_{0}}+\frac{\partial u_{2}}{\partial y_{1}}+\frac{\partial u_{7}}{\partial y_{4}}+\frac{\partial u_{4}}{\partial y_{7}} \\
& =\frac{\partial v_{1}}{\partial x_{2}}+\frac{\partial v_{0}}{\partial x_{3}}+\frac{\partial v_{6}}{\partial x_{5}}+\frac{\partial v_{5}}{\partial x_{6}}+\frac{\partial u_{1}}{\partial y_{2}}+\frac{\partial u_{0}}{\partial y_{3}}+\frac{\partial u_{6}}{\partial y_{5}}+\frac{\partial u_{5}}{\partial y_{6}}, \\
& \frac{\partial v_{4}}{\partial x_{0}}+\frac{\partial v_{6}}{\partial x_{2}}+\frac{\partial v_{7}}{\partial x_{3}}+\frac{\partial v_{0}}{\partial x_{4}}+\frac{\partial u_{4}}{\partial y_{0}}+\frac{\partial u_{6}}{\partial y_{2}}+\frac{\partial u_{7}}{\partial y_{3}}+\frac{\partial u_{0}}{\partial y_{4}} \\
& =\frac{\partial v_{5}}{\partial x_{1}}+\frac{\partial v_{1}}{\partial x_{5}}+\frac{\partial v_{2}}{\partial x_{6}}+\frac{\partial v_{3}}{\partial x_{7}}+\frac{\partial u_{5}}{\partial y_{1}}+\frac{\partial u_{1}}{\partial y_{5}}+\frac{\partial u_{2}}{\partial y_{6}}+\frac{\partial u_{3}}{\partial y_{7}},
\end{aligned}
$$




$$
\begin{aligned}
& \frac{\partial v_{5}}{\partial x_{0}}+\frac{\partial v_{4}}{\partial x_{1}}+\frac{\partial v_{6}}{\partial x_{3}}+\frac{\partial v_{3}}{\partial x_{6}}+\frac{\partial u_{5}}{\partial y_{0}}+\frac{\partial u_{4}}{\partial y_{1}}+\frac{\partial u_{6}}{\partial y_{3}}+\frac{\partial u_{3}}{\partial y_{6}} \\
= & \frac{\partial v_{7}}{\partial x_{2}}+\frac{\partial v_{1}}{\partial x_{4}}+\frac{\partial v_{0}}{\partial x_{5}}+\frac{\partial v_{2}}{\partial x_{7}}+\frac{\partial u_{7}}{\partial y_{2}}+\frac{\partial u_{1}}{\partial y_{4}}+\frac{\partial u_{0}}{\partial y_{5}}+\frac{\partial u_{2}}{\partial y_{7}} \\
& \frac{\partial v_{6}}{\partial x_{0}}+\frac{\partial v_{2}}{\partial x_{4}}+\frac{\partial v_{3}}{\partial x_{5}}+\frac{\partial v_{0}}{\partial x_{6}}+\frac{\partial u_{6}}{\partial y_{0}}+\frac{\partial u_{2}}{\partial y_{4}}+\frac{\partial u_{3}}{\partial y_{5}}+\frac{\partial u_{0}}{\partial y_{6}} \\
& =\frac{\partial v_{3}}{\partial x_{1}}+\frac{\partial v_{4}}{\partial x_{2}}+\frac{\partial v_{5}}{\partial x_{3}}+\frac{\partial v_{1}}{\partial x_{7}}+\frac{\partial u_{3}}{\partial y_{1}}+\frac{\partial u_{4}}{\partial y_{2}}+\frac{\partial u_{5}}{\partial y_{3}}+\frac{\partial u_{1}}{\partial y_{7}} \\
& \frac{\partial v_{7}}{\partial x_{0}}+\frac{\partial v_{6}}{\partial x_{1}}+\frac{\partial v_{5}}{\partial x_{2}}+\frac{\partial v_{2}}{\partial x_{5}}+\frac{\partial u_{7}}{\partial y_{0}}+\frac{\partial u_{6}}{\partial y_{1}}+\frac{\partial u_{5}}{\partial y_{2}}+\frac{\partial u_{2}}{\partial y_{5}} \\
= & \frac{\partial v_{4}}{\partial x_{3}}+\frac{\partial v_{3}}{\partial x_{4}}+\frac{\partial v_{1}}{\partial x_{6}}+\frac{\partial v_{0}}{\partial x_{7}}+\frac{\partial u_{7}}{\partial y_{0}}+\frac{\partial u_{6}}{\partial y_{1}}+\frac{\partial u_{5}}{\partial y_{2}}+\frac{\partial u_{2}}{\partial y_{5}} .
\end{aligned}
$$

Now, we add the following condition of integrability:

$$
\begin{aligned}
& \frac{\partial v_{0}}{\partial x_{0}}+\frac{\partial v_{3}}{\partial x_{3}}+\frac{\partial v_{5}}{\partial x_{5}}+\frac{\partial v_{7}}{\partial x_{7}}=\frac{\partial v_{1}}{\partial x_{1}}+\frac{\partial v_{2}}{\partial x_{2}}+\frac{\partial v_{4}}{\partial x_{4}}+\frac{\partial v_{6}}{\partial x_{6}} \\
& \frac{\partial v_{1}}{\partial x_{0}}+\frac{\partial v_{0}}{\partial x_{1}}+\frac{\partial v_{3}}{\partial x_{2}}+\frac{\partial v_{2}}{\partial x_{3}}+\frac{\partial v_{5}}{\partial x_{4}}+\frac{\partial v_{4}}{\partial x_{5}}+\frac{\partial v_{7}}{\partial x_{6}}+\frac{\partial v_{6}}{\partial x_{7}}=0, \\
& \frac{\partial v_{2}}{\partial x_{0}}+\frac{\partial v_{0}}{\partial x_{2}}+\frac{\partial v_{4}}{\partial x_{6}}+\frac{\partial v_{5}}{\partial x_{7}}=\frac{\partial v_{3}}{\partial x_{1}}+\frac{\partial v_{1}}{\partial x_{3}}+\frac{\partial v_{6}}{\partial x_{4}}+\frac{\partial v_{7}}{\partial x_{5}} \\
& \frac{\partial v_{3}}{\partial x_{0}}+\frac{\partial v_{2}}{\partial x_{1}}+\frac{\partial v_{7}}{\partial x_{4}}+\frac{\partial v_{4}}{\partial x_{7}}=\frac{\partial v_{1}}{\partial x_{2}}+\frac{\partial v_{0}}{\partial x_{3}}+\frac{\partial v_{6}}{\partial x_{5}}+\frac{\partial v_{5}}{\partial x_{6}} \\
& \frac{\partial v_{4}}{\partial x_{0}}+\frac{\partial v_{6}}{\partial x_{2}}+\frac{\partial v_{7}}{\partial x_{3}}+\frac{\partial v_{0}}{\partial x_{4}}=\frac{\partial v_{5}}{\partial x_{1}}+\frac{\partial v_{1}}{\partial x_{5}}+\frac{\partial v_{2}}{\partial x_{6}}+\frac{\partial v_{3}}{\partial x_{7}} \\
& \frac{\partial v_{5}}{\partial x_{0}}+\frac{\partial v_{4}}{\partial x_{1}}+\frac{\partial v_{6}}{\partial x_{3}}+\frac{\partial v_{3}}{\partial x_{6}}=\frac{\partial v_{7}}{\partial x_{2}}+\frac{\partial v_{1}}{\partial x_{4}}+\frac{\partial v_{0}}{\partial x_{5}}+\frac{\partial v_{2}}{\partial x_{7}} \\
& \frac{\partial v_{6}}{\partial x_{0}}+\frac{\partial v_{2}}{\partial x_{4}}+\frac{\partial v_{3}}{\partial x_{5}}+\frac{\partial v_{0}}{\partial x_{6}}=\frac{\partial v_{3}}{\partial x_{1}}+\frac{\partial v_{4}}{\partial x_{2}}+\frac{\partial v_{5}}{\partial x_{3}}+\frac{\partial v_{1}}{\partial x_{7}} \\
& \frac{\partial v_{7}}{\partial x_{0}}+\frac{\partial v_{6}}{\partial x_{1}}+\frac{\partial v_{5}}{\partial x_{2}}+\frac{\partial v_{2}}{\partial x_{5}}=\frac{\partial v_{4}}{\partial x_{3}}+\frac{\partial v_{3}}{\partial x_{4}}+\frac{\partial v_{1}}{\partial x_{6}}+\frac{\partial v_{0}}{\partial x_{7}}
\end{aligned}
$$

We let

$$
\begin{aligned}
d x y= & d x_{0} \wedge d x_{1} \wedge d x_{2} \wedge d x_{3} \wedge d x_{4} \wedge d x_{5} \wedge d x_{6} \wedge d x_{7} \\
& \wedge d y_{0} \wedge d y_{1} \wedge d y_{2} \wedge d y_{3} \wedge d y_{4} \wedge d y_{5} \wedge d y_{6} \wedge d y_{7}
\end{aligned}
$$

Theorem 3.1. Under the condition of integrability (3), let $F(z)$ be a hyperholomorphic function in an open set $\Omega$ of $\mathbb{C}^{4} \times \mathbb{C}^{4}$ and

$$
\begin{aligned}
& \kappa=d \hat{y}_{0}+e_{1} d \hat{y}_{1}+e_{2} d \hat{y}_{2}-e_{3} d \hat{y}_{3}+e_{4} d \hat{y}_{4}-e_{5} d \hat{y}_{5}+e_{6} d \hat{y}_{6}-e_{7} d \hat{y}_{7} \\
& +\varepsilon\left(d \hat{x}_{0}+e_{1} d \hat{x}_{1}+e_{2} d \hat{x}_{2}-e_{3} d \hat{x}_{3}+e_{4} d \hat{x}_{4}-e_{5} d \hat{x}_{5}+e_{6} d \hat{x}_{6}-e_{7} d \hat{x}_{7}\right),
\end{aligned}
$$


where $d \hat{x}_{j}$ is the $d x_{j}$-removed form on $d x y$, and $d \hat{y}_{j}$ is the $d y_{j}$-removed form on $d x y(j=0,1, \cdots, 7)$. Then for any domain $G \subset \Omega$ with smooth distinguished boundary $b G$,

$$
\int_{b G} \kappa F(z)=0
$$

where $\kappa F(z)$ is the product of dual octonions of the form $\kappa$ on the function $F(z)$.

Proof. By the rule of the multiplication of dual octonions, we have

$$
\begin{aligned}
& \kappa F(z)=\left(\hat{d y_{0}}+e_{1} d \hat{y}_{1}+e_{2} d \hat{y}_{2}-e_{3} d \hat{y}_{3}+e_{4} d \hat{y}_{4}-e_{5} d \hat{y}_{5}+e_{6} d \hat{y}_{6}-e_{7} d \hat{y}_{7}\right. \\
& +\varepsilon\left(d \hat{x}_{0}+e_{1} d \hat{x}_{1}+e_{2} d \hat{x}_{2}-e_{3} d \hat{x}_{3}+e_{4} d \hat{x}_{4}-e_{5} d \hat{x}_{5}+e_{6} d \hat{x}_{6}\right. \\
& \left.\left.-e_{7} d \hat{x}_{7}\right)\right) \cdot\left(\sum_{j=o}^{7} e_{j} u_{j}+\varepsilon \sum_{j=o}^{7} e_{j} v_{j}\right) \\
& =u_{o} d \hat{y}_{0}+e_{1} u_{1} d \hat{y}_{0}+e_{2} u_{2} d \hat{y}_{0}+e_{3} u_{3} d \hat{y}_{0}+e_{4} u_{4} d \hat{y}_{0}+e_{5} u_{5} d \hat{y}_{0} \\
& +e_{6} u_{6} d \hat{y}_{0}+e_{7} u_{7} d \hat{y}_{0}+e_{1} u_{0} d \hat{y}_{1}-u_{1} d \hat{y}_{1}+e_{3} u_{2} d \hat{y}_{1}-e_{2} u_{3} d \hat{y}_{1} \\
& +e_{5} u_{4} d \hat{y}_{1}-e_{4} u_{5} d \hat{y}_{1}+e_{7} u_{6} d \hat{y}_{1}-e_{6} u_{7} d \hat{y}_{1}+e_{2} u_{0} d \hat{y}_{2}-e_{3} u_{1} d \hat{y}_{2} \\
& -u_{2} d \hat{y}_{2}+e_{1} u_{3} d \hat{y}_{2}-e_{6} u_{4} d \hat{y}_{2}+e_{7} u_{5} d \hat{y}_{2}+e_{4} u_{6} d \hat{y}_{2}-e_{5} u_{7} d \hat{y}_{2} \\
& -e_{3} u_{0} d \hat{y}_{3}-e_{2} u_{1} d \hat{y}_{3}+e_{1} u_{2} d \hat{y}_{3}+u_{3} d \hat{y}_{3}-e_{7} u_{4} d \hat{y}_{3}-e_{6} u_{5} d \hat{y}_{3} \\
& +e_{5} u_{6} d \hat{y}_{3}+e_{4} u_{7} d \hat{y}_{3}+e_{4} u_{0} d \hat{y}_{4}-e_{5} u_{1} d \hat{y}_{4}+e_{6} u_{2} d \hat{y}_{4}-e_{7} u_{3} d \hat{y}_{4} \\
& -u_{4} d \hat{y}_{4}+e_{1} u_{5} d \hat{y}_{4}-e_{2} u_{6} d \hat{y}_{4}+e_{3} u_{7} d \hat{y}_{4}-e_{5} u_{0} d \hat{y}_{5}-e_{4} u_{1} d \hat{y}_{5} \\
& +e_{7} u_{2} d \hat{y}_{5}+e_{6} u_{3} d \hat{y}_{5}+e_{1} u_{4} d \hat{y}_{5}+u_{5} d \hat{y}_{5}-e_{3} u_{6} d \hat{y}_{5}-e_{2} u_{7} d \hat{y}_{5} \\
& +e_{6} u_{0} d \hat{y}_{6}-e_{7} u_{1} d \hat{y}_{6}-e_{4} u_{2} d \hat{y}_{6}+e_{5} u_{3} d \hat{y}_{6}+e_{2} u_{4} d \hat{y}_{6}-e_{3} u_{5} d \hat{y}_{6} \\
& -u_{6} d \hat{y}_{6}+e_{1} u_{7} d \hat{y}_{6}-e_{7} u_{0} d \hat{y}_{7}-e_{6} u_{1} d \hat{y}_{7}-e_{5} u_{2} d \hat{y}_{7}-e_{4} u_{3} d \hat{y}_{7} \\
& +e_{3} u_{4} d \hat{y}_{7}+e_{2} u_{5} d \hat{y}_{7}+e_{1} u_{6} d \hat{y}_{7}+u_{7} d \hat{y}_{7}+\varepsilon\left(v_{0} d \hat{y}_{0}+e_{1} v_{1} d \hat{y}_{0}\right. \\
& +e_{2} v_{2} d \hat{y}_{0}+e_{3} v_{3} d \hat{y}_{0}+e_{4} v_{4} d \hat{y}_{0}+e_{5} v_{5} d \hat{y}_{0}+e_{6} v_{6} d \hat{y}_{0}+e_{7} v_{7} d \hat{y}_{0} \\
& +e_{1} v_{0} d \hat{y}_{1}-v_{1} d \hat{y}_{1}+e_{3} v_{2} d \hat{y}_{1}-e_{2} v_{3} d \hat{y}_{1}+e_{5} v_{4} d \hat{y}_{1}-e_{4} v_{5} d \hat{y}_{1} \\
& +e_{7} v_{6} d \hat{y}_{1}-e_{6} v_{7} d \hat{y}_{1}+e_{2} v_{0} d \hat{y}_{2}-e_{3} v_{1} d \hat{y}_{2}-v_{2} d \hat{y}_{2}+e_{1} v_{3} d \hat{y}_{2} \\
& -e_{6} v_{4} d \hat{y}_{2}+e_{7} v_{5} d \hat{y}_{2}+e_{4} v_{6} d \hat{y}_{2}-e_{5} v_{7} d \hat{y}_{2}-e_{3} v_{0} d \hat{y}_{3}-e_{2} v_{1} d \hat{y}_{3} \\
& +e_{1} v_{2} d \hat{y}_{3}+v_{3} d \hat{y}_{3}-e_{7} v_{4} d \hat{y}_{3}-e_{6} v_{5} d \hat{y}_{3}+e_{5} v_{6} d \hat{y}_{3}+e_{4} v_{7} d \hat{y}_{3} \\
& +e_{4} v_{0} d \hat{y}_{4}-e_{5} v_{1} d \hat{y}_{4}+e_{6} v_{2} d \hat{y}_{4}-e_{7} v_{3} d \hat{y}_{4}-v_{4} d \hat{y}_{4}+e_{1} v_{5} d \hat{y}_{4} \\
& -e_{2} v_{6} d \hat{y}_{4}+e_{3} v_{7} d \hat{y}_{4}-e_{5} v_{0} d \hat{y}_{5}-e_{4} v_{1} d \hat{y}_{5}+e_{7} v_{2} d \hat{y}_{5}+e_{6} v_{3} d \hat{y}_{5} \\
& +e_{1} v_{4} d \hat{y}_{5}+v_{5} d \hat{y}_{5}-e_{3} v_{6} d \hat{y}_{5}-e_{2} v_{7} d \hat{y}_{5}+e_{6} v_{0} d \hat{y}_{6}-e_{7} v_{1} d \hat{y}_{6} \\
& -e_{4} v_{2} d \hat{y}_{6}+e_{5} v_{3} d \hat{y}_{6}+e_{2} v_{4} d \hat{y}_{6}-e_{3} v_{5} d \hat{y}_{6}-v_{6} d \hat{y}_{6}+e_{1} v_{7} d \hat{y}_{6} \\
& -e_{7} v_{0} d \hat{y}_{7}-e_{6} v_{1} d \hat{y}_{7}-e_{5} v_{2} d \hat{y}_{7}-e_{4} v_{3} d \hat{y}_{7}+e_{3} v_{4} d \hat{y}_{7}+e_{2} v_{5} d \hat{y}_{7}
\end{aligned}
$$




$$
\begin{aligned}
& +e_{1} v_{6} d \hat{y}_{7}+v_{7} d \hat{y}_{7}+u_{o} d \hat{x}_{0}+e_{1} u_{1} d \hat{x}_{0}+e_{2} u_{2} d \hat{x}_{0}+e_{3} u_{3} d \hat{x}_{0} \\
& +e_{4} u_{4} d \hat{x}_{0}+e_{5} u_{5} d \hat{x}_{0}+e_{6} u_{6} d \hat{x}_{0}+e_{7} u_{7} d \hat{x}_{0}+e_{1} u_{0} d \hat{x}_{1}-u_{1} d \hat{x}_{1} \\
& +e_{3} u_{2} d \hat{x}_{1}-e_{2} u_{3} d \hat{x}_{1}+e_{5} u_{4} d \hat{x}_{1}-e_{4} u_{5} d \hat{x}_{1}+e_{7} u_{6} d \hat{x}_{1}-e_{6} u_{7} d \hat{x}_{1} \\
& +e_{2} u_{0} d \hat{x}_{2}-e_{3} u_{1} d \hat{x}_{2}-u_{2} d \hat{x}_{2}+e_{1} u_{3} d \hat{x}_{2}-e_{6} u_{4} d \hat{x}_{2}+e_{7} u_{5} d \hat{x}_{2} \\
& +e_{4} u_{6} d \hat{x}_{2}-e_{5} u_{7} d \hat{x}_{2}-e_{3} u_{0} d \hat{x}_{3}-e_{2} u_{1} d \hat{x}_{3}+e_{1} u_{2} d \hat{x}_{3}+u_{3} d \hat{x}_{3} \\
& -e_{7} u_{4} d \hat{x}_{3}-e_{6} u_{5} d \hat{x}_{3}+e_{5} u_{6} d \hat{x}_{3}+e_{4} u_{7} d \hat{x}_{3}+e_{4} u_{0} d \hat{x}_{4}-e_{5} u_{1} d \hat{x}_{4} \\
& +e_{6} u_{2} d \hat{x}_{4}-e_{7} u_{3} d \hat{x}_{4}-u_{4} d \hat{x}_{4}+e_{1} u_{5} d \hat{x}_{4}-e_{2} u_{6} d \hat{x}_{4}+e_{3} u_{7} d \hat{x}_{4} \\
& -e_{5} u_{0} d \hat{x}_{5}-e_{4} u_{1} d \hat{x}_{5}+e_{7} u_{2} d \hat{x}_{5}+e_{6} u_{3} d \hat{x}_{5}+e_{1} u_{4} d \hat{x}_{5}+u_{5} d \hat{x} 5 \\
& -e_{3} u_{6} d \hat{x}_{5}-e_{2} u_{7} d \hat{x}_{5}+e_{6} u_{0} d \hat{x}_{6}-e_{7} u_{1} d \hat{x}_{6}-e_{4} u_{2} d \hat{x}_{6}+e_{5} u_{3} d \hat{x}_{6} \\
& +e_{2} u_{4} d \hat{x}_{6}-e_{3} u_{5} d \hat{x}_{6}-u_{6} d \hat{x}_{6}+e_{1} u_{7} d \hat{x}_{6}-e_{7} u_{0} d \hat{x}_{7}-e_{6} u_{1} d \hat{x}_{7} \\
& \left.-e_{5} u_{2} d \hat{x}_{7}-e_{4} u_{3} d \hat{x}_{7}+e_{3} u_{4} d \hat{x}_{7}+e_{2} u_{5} d \hat{x}_{7}+e_{1} u_{6} d \hat{x}_{7}+u_{7} d \hat{x}_{7}\right) .
\end{aligned}
$$

Therefore,

$$
\begin{aligned}
d(\kappa F)= & \left(\sum_{j=o}^{7} \frac{\partial}{\partial x_{j}} d x_{j}+\varepsilon \sum_{j=o}^{7} \frac{\partial}{\partial y_{j}} d y_{j}\right)(\kappa F) \\
= & \varepsilon\left(\left(\frac{\partial u_{0}}{\partial y_{0}}-\frac{\partial u_{1}}{\partial y_{1}}-\frac{\partial u_{2}}{\partial y_{2}}+\frac{\partial u_{3}}{\partial y_{3}}-\frac{\partial u_{4}}{\partial y_{4}}+\frac{\partial u_{5}}{\partial y_{5}}-\frac{\partial u_{6}}{\partial y_{6}}+\frac{\partial u_{7}}{\partial y_{7}}\right.\right. \\
& \left.+\frac{\partial u_{0}}{\partial x_{0}}-\frac{\partial u_{1}}{\partial x_{1}}-\frac{\partial u_{2}}{\partial x_{2}}+\frac{\partial u_{3}}{\partial x_{3}}-\frac{\partial u_{4}}{\partial x_{4}}+\frac{\partial u_{5}}{\partial x_{5}}-\frac{\partial u_{6}}{\partial x_{6}}+\frac{\partial u_{7}}{\partial x_{7}}\right) d x y \\
& +e_{1}\left(\frac{\partial u_{1}}{\partial y_{0}}+\frac{\partial u_{0}}{\partial y_{1}}+\frac{\partial u_{3}}{\partial y_{2}}+\frac{\partial u_{2}}{\partial y_{3}}+\frac{\partial u_{5}}{\partial y_{4}}+\frac{\partial u_{4}}{\partial y_{5}}+\frac{\partial u_{7}}{\partial y_{6}}+\frac{\partial u_{6}}{\partial y_{7}}\right. \\
& \left.+\frac{\partial u_{1}}{\partial x_{0}}+\frac{\partial u_{0}}{\partial x_{1}}+\frac{\partial u_{3}}{\partial x_{2}}+\frac{\partial u_{2}}{\partial x_{3}}+\frac{\partial u_{5}}{\partial x_{4}}+\frac{\partial u_{4}}{\partial x_{5}}+\frac{\partial u_{7}}{\partial x_{6}}+\frac{\partial u_{6}}{\partial x_{7}}\right) d x y \\
& +e_{2}\left(\frac{\partial u_{2}}{\partial y_{0}}-\frac{\partial u_{3}}{\partial y_{1}}+\frac{\partial u_{0}}{\partial y_{2}}-\frac{\partial u_{1}}{\partial y_{3}}-\frac{\partial u_{6}}{\partial y_{4}}-\frac{\partial u_{7}}{\partial y_{5}}+\frac{\partial u_{4}}{\partial y_{6}}+\frac{\partial u_{5}}{\partial y_{7}}\right. \\
& \left.+\frac{\partial u_{2}}{\partial x_{0}}-\frac{\partial u_{3}}{\partial x_{1}}+\frac{\partial u_{0}}{\partial x_{2}}-\frac{\partial u_{1}}{\partial x_{3}}-\frac{\partial u_{6}}{\partial x_{4}}-\frac{\partial u_{7}}{\partial x_{5}}+\frac{\partial u_{4}}{\partial x_{6}}+\frac{\partial u_{5}}{\partial x_{7}}\right) d x y \\
& +e_{3}\left(\frac{\partial u_{3}}{\partial y_{0}}+\frac{\partial u_{2}}{\partial y_{1}}-\frac{\partial u_{1}}{\partial y_{2}}-\frac{\partial u_{0}}{\partial y_{3}}+\frac{\partial u_{7}}{\partial y_{4}}-\frac{\partial u_{6}}{\partial y_{5}}-\frac{\partial u_{5}}{\partial y_{6}}+\frac{\partial u_{4}}{\partial y_{7}}\right. \\
& \left.+\frac{\partial u_{3}}{\partial x_{0}}+\frac{\partial u_{2}}{\partial x_{1}}-\frac{\partial u_{1}}{\partial x_{2}}-\frac{\partial u_{0}}{\partial x_{3}}+\frac{\partial u_{7}}{\partial x_{4}}-\frac{\partial u_{6}}{\partial x_{5}}-\frac{\partial u_{5}}{\partial x_{6}}+\frac{\partial u_{4}}{\partial x_{7}}\right) d x y \\
& +e_{4}\left(\frac{\partial u_{4}}{\partial y_{0}}-\frac{\partial u_{5}}{\partial y_{1}}+\frac{\partial u_{6}}{\partial y_{2}}+\frac{\partial u_{7}}{\partial y_{3}}+\frac{\partial u_{0}}{\partial y_{4}}-\frac{\partial u_{1}}{\partial y_{5}}-\frac{\partial u_{2}}{\partial y_{6}}-\frac{\partial u_{3}}{\partial y_{7}}\right. \\
& \left.+\frac{\partial u_{4}}{\partial x_{0}}-\frac{\partial u_{5}}{\partial x_{1}}+\frac{\partial u_{6}}{\partial x_{2}}+\frac{\partial u_{7}}{\partial x_{3}}+\frac{\partial u_{0}}{\partial x_{4}}-\frac{\partial u_{1}}{\partial x_{5}}-\frac{\partial u_{2}}{\partial x_{6}}-\frac{\partial u_{3}}{\partial x_{7}}\right) d x y \\
&
\end{aligned}
$$




$$
\begin{aligned}
& +e_{5}\left(\frac{\partial u_{5}}{\partial y_{0}}+\frac{\partial u_{4}}{\partial y_{1}}-\frac{\partial u_{7}}{\partial y_{2}}+\frac{\partial u_{6}}{\partial y_{3}}-\frac{\partial u_{1}}{\partial y_{4}}-\frac{\partial u_{0}}{\partial y_{5}}+\frac{\partial u_{3}}{\partial y_{6}}-\frac{\partial u_{2}}{\partial y_{7}}\right. \\
& \left.+\frac{\partial u_{5}}{\partial x_{0}}+\frac{\partial u_{4}}{\partial x_{1}}-\frac{\partial u_{7}}{\partial x_{2}}+\frac{\partial u_{6}}{\partial x_{3}}-\frac{\partial u_{1}}{\partial x_{4}}-\frac{\partial u_{0}}{\partial x_{5}}+\frac{\partial u_{3}}{\partial x_{6}}-\frac{\partial u_{2}}{\partial x_{7}}\right) d x y \\
& +e_{6}\left(\frac{\partial u_{6}}{\partial y_{0}}-\frac{\partial u_{7}}{\partial y_{1}}-\frac{\partial u_{4}}{\partial y_{2}}-\frac{\partial u_{5}}{\partial y_{3}}+\frac{\partial u_{2}}{\partial y_{4}}+\frac{\partial u_{3}}{\partial y_{5}}+\frac{\partial u_{0}}{\partial y_{6}}-\frac{\partial u_{1}}{\partial y_{7}}\right. \\
& \left.+\frac{\partial u_{6}}{\partial x_{0}}-\frac{\partial u_{7}}{\partial x_{1}}-\frac{\partial u_{4}}{\partial x_{2}}-\frac{\partial u_{5}}{\partial x_{3}}+\frac{\partial u_{2}}{\partial x_{4}}+\frac{\partial u_{3}}{\partial x_{5}}+\frac{\partial u_{0}}{\partial x_{6}}-\frac{\partial u_{1}}{\partial x_{7}}\right) d x y \\
& +e_{7}\left(\frac{\partial u_{7}}{\partial y_{0}}+\frac{\partial u_{6}}{\partial y_{1}}+\frac{\partial u_{5}}{\partial y_{2}}-\frac{\partial u_{4}}{\partial y_{3}}-\frac{\partial u_{3}}{\partial y_{4}}+\frac{\partial u_{2}}{\partial y_{5}}-\frac{\partial u_{1}}{\partial y_{6}}-\frac{\partial u_{0}}{\partial y_{7}}\right. \\
& \left.\left.+\frac{\partial u_{7}}{\partial x_{0}}+\frac{\partial u_{6}}{\partial x_{1}}+\frac{\partial u_{5}}{\partial x_{2}}-\frac{\partial u_{4}}{\partial x_{3}}-\frac{\partial u_{3}}{\partial x_{4}}+\frac{\partial u_{2}}{\partial x_{5}}-\frac{\partial u_{1}}{\partial x_{6}}-\frac{\partial u_{0}}{\partial x_{7}}\right) d x y\right) .
\end{aligned}
$$

By Equations (2) and (3), we have $d(\kappa F)=0$. By Stokes theorem, we have

$$
\int_{b G} \kappa F=\int_{G} d(\kappa F)=0 .
$$

\section{References}

[1] P. Dentoni and M. Sce, Funzioni regolari nell'algebra di Cayley, Rend. Sem. Mat. Univ. Pador 50 (1973), 251-267.

[2] H. S. Jung, S. J. Ha, K. H. Lee, S. M. Lim and K. H. Shon, Structures of hyperholomorphic functions on dual quaternion numbers, Honam Mathematical J. 35 (2013), 809-817.

[3] S. J. Lim and K. H. Shon, Hyperholomorphic functions and hyperconjugate harmonic functions of octonion variables, J. Inequa. Appl. 77 (2013), 1-8.

[4] K. Nôno and H. Koriyama, On regularities of octonionic functions and holomorphic mappings, Bull. Fukuoka Univ. Education 60 part III (2011), 11-28.

[5] K. Nôno, Hyperholomorphic functions of a quaternion variable, Bull. Fukuoka Univ. Ed. 32 (1983), 21-37.

[6] K. Nôno, On the octonionic linearization of Laplacian and octonionic function theory, Bull. Fukuoka Univ. Ed. 37 (1988), 1-15.

Su Mi LIM

Department of Mathematics, Pusan National University, Busan 609-735, Korea

E-mail address: aiqiqiq@naver.com

KWANG Ho SHON

Department of Mathematics, Pusan National University, Busan 609-735, Korea

E-mail address: khshon@pusan.ac.kr 